partly coagulated at the positive pole. At the cathode they are partly changed into alkali-albumin.

Bile and urine were taken as further examples of animal liquids.

(4) The effect of electrolysis in the living body.

Pithed frogs and anæsthetized rabbits were used. This part of the work is still incomplete.

\section{G. N. StEWART.}

\section{LOBSTER CULTURE IN THE ISLE OF MULL.}

$\mathrm{W}^{\mathrm{E}}$ have been favoured with a circular, issued by Mr. George Brook, Lecturer on Embryology in the University of Edinburgh, and Mr. W. L. Calderwood, late of the scientific staff of the Fishery Board for Scotland, expressive of an intentention to establish at Lochbuie a small marine laboratory. The promoters have set themselves to restore our shell fisheries to their former condition; and a leading item in their programme is the proposal to construct a lobster pond, with suitable apparatus for hatching and rearing lobsters. The cost of the entire laboratory, with pond and plant, is estimated at $£ 400$, that of maintenance at $\ell_{150}$ per annum-exceedingly moderate sums, for which an appeal is made to the public. The condition into which our lobster fisheries have lapsed is shown by the fact that a lobster ground in the far west of Ireland is worked by a South of England boat. Our import lobster trade is yearly increasing, and the fact that our markets are not home-stocked is discreditable in the extreme. The problem of artificial culture necessary for the purpose in view has many times been attacked by British naturalists. Saville Kent had it constantly in mind while officiating at our several aquaria ; he made it a primary object in his schemes for the establishment of marine stations in Jersey and at Brighton, and he meanwhile attempted to raise interest in it in a paper read at the International Fisheries Exhibition held at South Kensington. All this notwithstanding, the matter has, with us, not yet passed beyond the experimental stage, and we are behind in the international race. At Lochbuie the conditions should be favourable; and as Mr. Brook, in the preparation of his Challenger Report, has shown himself capable of performing a difficult task under exceptional conditions, we have full confidence in his ability to carry out his project. The promoters of this scheme propose in other respects to pursue a course of scientific study of the marine fauna of the west coast of Scotland, but their chief aims are unmistakably economic. We sincerely hope that they will confine their attention to the one or the other branch, for nothing can be plainer than that the extraordinary successes which have placed the fishery work of our American cousins foremost in that of the world, have been largely, if not wholly, due to their having kept pure science and economics scrupulously apart. The Lochbuie scheme is a modest though an ambitious one, and Messrs. Brook and Calderwood signify their intention of giving their services as superintendents. Recent proceedings in Parliament have shown that there is disaffection on the Scottish Fishery Board ; and it would be an interesting circumstance should private enterprise, which has done so much for science in Britain, solve the difficulty in hand, while the State-aided body fritters away a handsome endowment.

\section{THE FRENCH ASSOCIATION FOR THE $A D V A N C E M E N T$ OF SCIENCE.}

$T \mathrm{HE}$ nineteenth meeting of the French Association for the Advancement of Science opened at Limoges on the 7 th inst.

$$
\text { NO. IO86, VOL. } 42]
$$

After some remarks on the learned societies of Limoges. and some references to Gay-Lussac, the inauguration of whose statue took place on the I Ith inst., Prof. A. Cornu, the President of the Association for the year, delivered a discourse on the part played by physics in the progress of the sciences. It is impossible in the space at our disposal to do justice to this interesting address, but the following will give an idea of its character.

Beginning with chemistry, Prof. Cornu pointed out that the introduction and use of the chemical balance by Richter, Wenzel, Dalton, and Lavoisier led to the substitution of the laws of multiple and equivalent proportions, and the indestructibility of matter, for the vague hypotheses held by the alchemists.

Two other physical instruments introduced into chemical methods are the calorimeter and barometer. By means of the first, Dulong and Petit's law, that the same quantity of heat is required to heat an atom of all simple bodies to the same extent, was discovered ; and but for the second, Gay-Lussac could not have made his researches on vapour density, which, with the work of Amperre and Avogadro, led to the determination of the numerical relation between the temperature density and pressure of a gas and the notion of atomic volume.

Another common physical instrument, the thermometer, has furnished organic chemistry with the means of discovering important laws of organic series; and recently, with the calorimeter, it has enabled $M$. Rault to determine molecular weights by the freezing of dissolvents, and has furnished Thomson, Berthelot, Sarrau, Vielle, and other workers in thermo-chemistry with the means of arriving at the new mechanics of the affinity of atoms according to their size, like the universal law of gravitation.

The introduction of the spectroscope into the chemical laboratory for purposes of analysis, by Bunsen and Kirchoff, marks an important epoch in the history of chemistry. This instrument has been entirely created by the labours of physicists ; the prism of Newton, the telescope of Fraunhofer, and the collimator of Babinet marking stages in its evolution Bunsen and Kirchoff demonstrated the power of their method of analysis by the discovery of rubidium and cæsium; in fact, it is only necessary to observe an unknown line in the spectrum of a substance to establish the existence of a new element.

It appears therefore that each time chemistry has borrowed from physics some new method it has entered into a prolific field of investigation, conceptions have been extended and given a more precise meaning, and chemical knowledge advanced in a manner proportional to the power of the adopted methods.

The other natural sciences have benefited in the same way. Up to the seventeenth century astronomers had no means of assisting their vision, and therefore they could only make observations of the movements of the heavenly bodies. In spite, however, of the simplicity of the means of observation, the work of Hipparchus, Ptolemy, Copernicus, Tycho-Brahé, and Kepler contained a considerable amount of information with respect to celestial motions, but nothing was known of the constitution of the bodies observed. With the refracting telescope of Galileo and Newton's reflector, astronomy underwent a transformation: the sun was found to have spots and faculæ; the plains, mountains, and craters of the moon were observed: Venus was shown to go through phases in the same manner as our satellite; Jupiter's belts and satellites were seen; and the beauty of Saturn and his rings revealed.

Later, Herschel's large mirrors, worked by his own hands, enabled him to discover double and multiple star systems ; to prove that many stars are suns like our own, inasmuch as they have other bodies revolving round them.

Such was the revolution produced in astronomy by the employment of the first optical instruments. The intro- 
duction of the spectroscope considerably extended the limits of investigation. The chemical constitution of the stars was determined in spite of their immense distances ; the sun was shown to contain sodium, iron, magnesium, calcium, hydrogen, in a state of vapour at its surface-that is, the same elements as those which make up the earth's crust ; it also contains nickel, an important constituent of meteorites, those nomadic bodies which fill interplanetary space. The sun and the bodies revolving round it are therefore composed of the same elements.

By means of the spectroscope it has been proved that the moon and the planets shine by reflected light, and that the stars, like the sun, are self-luminous, and made up of the same elements, thus demonstrating the unity of the chemical composition of the whole universe.

But the spectroscope has not only revealed the substance of the stellar world, it affords a means of investigating a component of stellar motion. The principle enunciated by Doppler, viz. that light-waves, like those of sound, vary in length with the relative velocity of the source producing it, remained unapplied for some time because there was, of course, no means of determining the proper colour of a star in repose and comparing it with that received, the variation being produced by motion in the line of sight. Fizeau showed, however, that by substituting lines in the spectrum for the idea of colour the conditions necessary for the application of the principle were met ; all that was required being a line common to a star and some terrestrial element, and the measurement of the displacement of this line. This method was proposed by Fizeau in 1859 , and has been considerably developed; numerous lines in stellar spectra are coincident with those of terrestrial substances. If they are all shifted towards the red the star is receding from the earth ; if towards the violet the star is approaching us. The displacement of the line is measured with a micrometer, and a simple calculation gives the velocity with which the star is moving, whatever may be its distance.

It has been shown that for the application of the Doppler-Fizeau principle it is necessary to find in the spectrum of the star the lines of a terrestrial element. This common element is most often hydrogen - the simple body par excellence, the elementary substance of those who hold in the unity of matter.

Among all the methods of rendering impurities manifest, the simplest and most delicate is that of spectrum analysis. With the spectrum of hydrogen observed in the laboratory feeble lines of other substances are always present, and to decide upon the true hydrogen spectrum becomes therefore a difficult matter. But it was an astronomer and not a chemist who first described the pure hydrogen spectrum; the lines photographed by Dr. Huggins in the spectra of the white stars having since been shown to be reproduced in the laboratory when the spectrum of approximately pure hydrogen is observed.

In physics, the centre of natural philosophy, many branches have made rapid and definite advances. The results of the development of electrical science is seen on all sides, yet no science has had a more humble beginning. The first electrical experiment was made six centuries before our era: this was the attraction of light bodies by rubbed amber. The knowledge remained in this stage for more than twenty centuries; then the two electrical states were gradually recognized, and conductors and non-conductors were separated. In the establishment of the identity of atmospheric electricity with that obtained by electrical machines the death of Richmann at St. Petersburg should be noticed, and the discovery of the lightning conductor by the illustrious Franklin.

Everyone knows the story of the convulsive movements of a frog's leg in contact with a bimetallic arc observed by Galvani, an Italian physiologist. Volta saw in this circumstance that electricity might be developed by the contact of different substances; he discovered the law NO. IO 86 , VOL. 42 ] which permitted the energy to be multiplied ; and in 1794 summed up all his works in an imperishable monument -the voltaic pile.

All the sciences benefited by the discovery, but chemistry gained the most. Carlisle and Nicholson decomposed water; Davy, with the great pile belonging to the Royal Institution of London, decomposed the alkalies and alkaline earths, formerly supposed elementary bodies. Later, Davy performed an experiment which eclipsed everything accomplished with the invention of Volta. By joining two carbon poles to his colossal pile, he produced a dazzling and continuous light, and discovered the electric arc now so commonly seen.

In 1820 , Oersted discovered that the wire joining the poles of a pile exercised an influence on a magnetic needle. Ampère discovered the mutual action of electric currents, the mathematical law governing it, and, finally, the production of magnetism by the sole action of the voltaic current.

The discovery of the electro-magnet was a great event, not only in the history of science, but in that of humanity. In telegraphy it is the electro-magnet which transmits messages from one end of the world to the other with the velocity of light; in the telephone, the word itself; in the powerful machines derived from the memorable discoveries of Faraday, it is that which causes the transformation of energy.

Great advancements have also been made on the purely theoretical side. Ampère, Poisson, Fourier, Ohm, Gauss, Helmholtz, Thomson, and Maxwell have done much to connect electricity with mechanical laws. Again, electro-magnetic and optical phenomena obey the same elementary laws, and appear to be two manifestations of the movement of the same medium-the ether; thus optical problems may be settled with the equations of electro-magnetism. From an experimental point of view, results full of promise have already been obtained; the velocity of light, found by optical methods, has also been determined by measures purely electrical; and recently $M$. Hertz has accomplished experimentally the identification of electrical discharges with lightwaves.

All these facts show that as our knowledge increases the distinctions between different branches of science vanish the limits which have been traced between them are shown to be artificial, and only testify to ignorance of natural laws; but the efforts of successive generations have not been in vain, and we look forward to the time when these limits will be effaced, and all the branches of natural philosophy be united in one harmonious whole.

Prof. Cornu's discourse, of which the foregoing is but a sketch, was received with much applause.

After an address by the Mayor of Limoges, the Secretary of the Association, M. A. Gobin, read the report for I 889-90, and gave an account of the meeting in Paris last year. The financial statement by M. Gallante shows that the Association is in a prosperous condition, and increasing its number of members.

Many interesting and important communications were made in the different sections. The series of excursions included one of three days' duration, and visits were made to all places of interest in or near Limoges. The Congress will be remembered as a very successful one by all who were fortunate enough to be present.

\section{H. F. PETERS.}

BY the death of Prof. C. H. F. Peters, Director of the Litchfield Observatory, Hamilton College, Clinton, N.Y.; astronomy has lost an assiduous observer. An in teresting sketch of his career is given in the Astronomische Nachrichten, from which the following details are taken. 